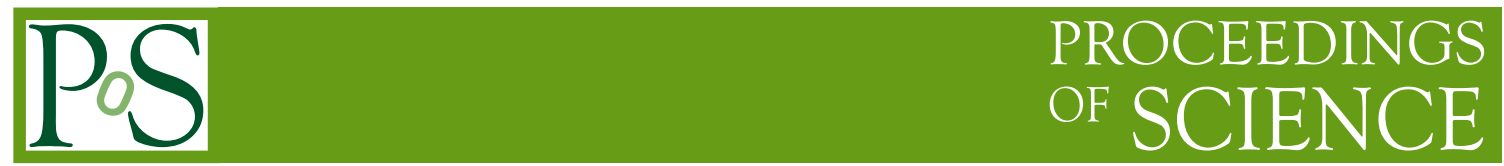

\title{
Optimization of Beam Line Settings for MICE Step IV
}

\author{
John Columba Nugent* \\ University of Glasgow \\ E-mail: j.nugent.1@research.gla.ac.uk \\ Jaroslaw Pasternak \\ Imperial College London \\ E-mail: j.pasternak@imperial.ac.uk
}

The Muon Ionization Cooling Experiment (MICE) is progressing towards the first demonstration of ionization cooling in 2017-18. Construction of MICE Step IV will be completed in 2015. Before data taking, an essential part of the commissioning will be determining the beam line settings which produce a matched beam in MICE. Monte Carlo data from MICE was generated with G4beamline and nominal settings for the beam were produced.

16th International Workshop on Neutrino Factories and Future Neutrino Beam Facilities 25 -30 August, 2014

University of Glasgow, United Kingdom

\footnotetext{
* Speaker.
} 


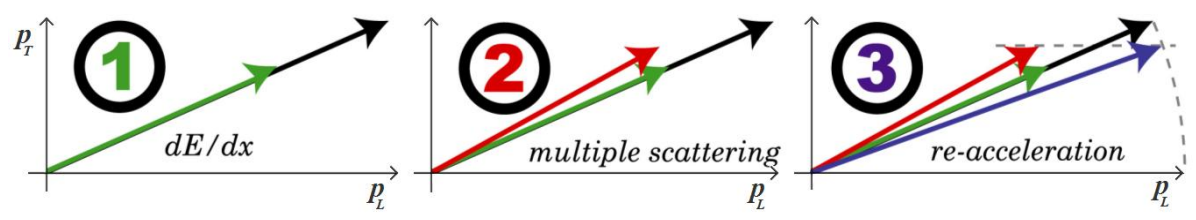

Figure 1: Reduction of phase-space volume of muon beam.

\section{Introduction}

Results from atmospheric neutrinos at Super-Kamiokande [1] and from solar neutrinos at the Sudbury Neutrino Observatory [2] conclusively demonstrated that neutrinos have a non-zero mass and oscillate between different flavours. A facility promising precision measurement of neutrino oscillations parameters is the Neutrino Factory [3], where neutrinos would be produced via muon decay rings. Before the muons are injected into the storage ring the phase-space volume of the beam must be reduced. The only cooling technique which can act within the lifetime of the muon is ionization cooling. MICE is progressing towards a demonstration of this technology with Step IV providing the first measurement of ionization cooling. Demonstration of this technology is an essential part of the worldwide research effort towards building a Neutrino Factory. A Neutrino Factory is the only proposed facility with the capability to measure the CP violation phase, $\delta_{C P}$, with $5^{\circ}$ accuracy.

\section{Ionization Cooling}

Ionization cooling results from the combination of an energy loss by ionization as the beam passes through a material and RF accelerating cavities, complemented by magnetic focusing. A muon beam crossing an absorber loses energy by ionization reducing the longitudinal and transverse momenta of the muons. A high gradient RF cavity is used to restore the longitudinal momentum of the beam (figure 1). The rate of change of normalized emittance in a medium of thickness $\mathrm{X}$ may be described as [4]:

$$
\frac{d \varepsilon_{N}}{d X} \approx-\frac{\varepsilon_{N}}{\beta^{2} E_{\mu}}\left\langle\frac{d E}{d X}\right\rangle+\frac{\beta_{\perp}(0.014 G e V)^{2}}{2 \beta^{3} E_{\mu} m_{\mu} X_{0}} ;
$$

where $\varepsilon_{N}$ is the normalized transverse (2-dimensional) emittance of the beam, $X_{0}$ is the radiation length of the medium, $\beta_{\perp}$ is the betatron function (in a description of the beam in terms of the Twiss parameters $\beta_{\perp}, \alpha_{\perp}, \gamma_{\perp}$ and the phase function $\left.\psi_{\perp}\right), E_{\mu}$ and $m_{\mu}$ the energy and mass of the muon and $\beta=p c / E$. The first term on the right hand side of equation 1 describes the cooling effect and the second the heating effect, due to multiple scattering. The emittance of the beam when these two effects are at equilibrium is:

$$
\varepsilon_{e q} \approx \frac{\beta_{\perp}(0.014 G e V)^{2}}{2 \beta m_{\mu} X_{0}}\left\langle\frac{d E}{d X}\right\rangle^{-1} .
$$

The lower the equilibrium emittance the better the cooling channel. To achieve this, $\beta_{\perp}$ should be minimised which requires strong focusing at the absorber and $X_{0}\left\langle\frac{d E}{d X}\right\rangle$ should be maximised. 


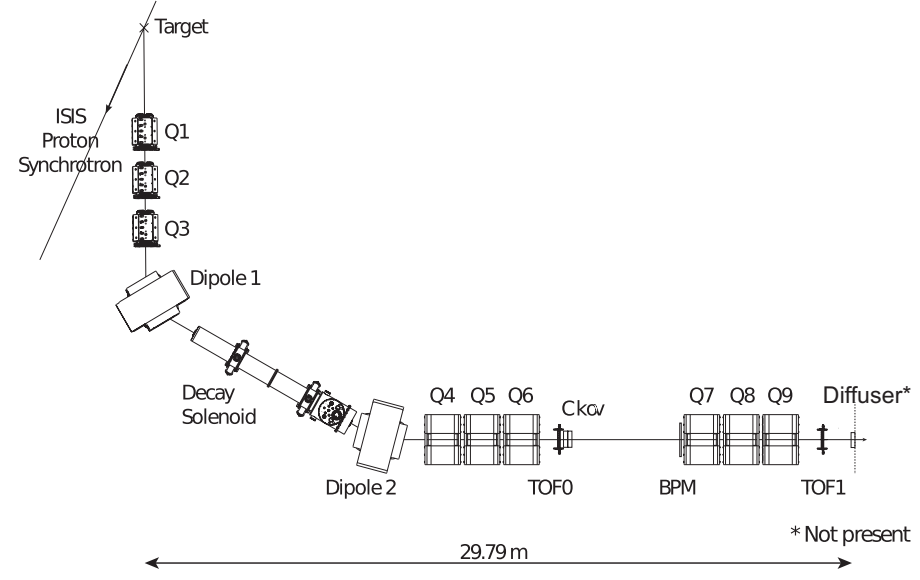

Figure 2: Layout of the MICE beam line.
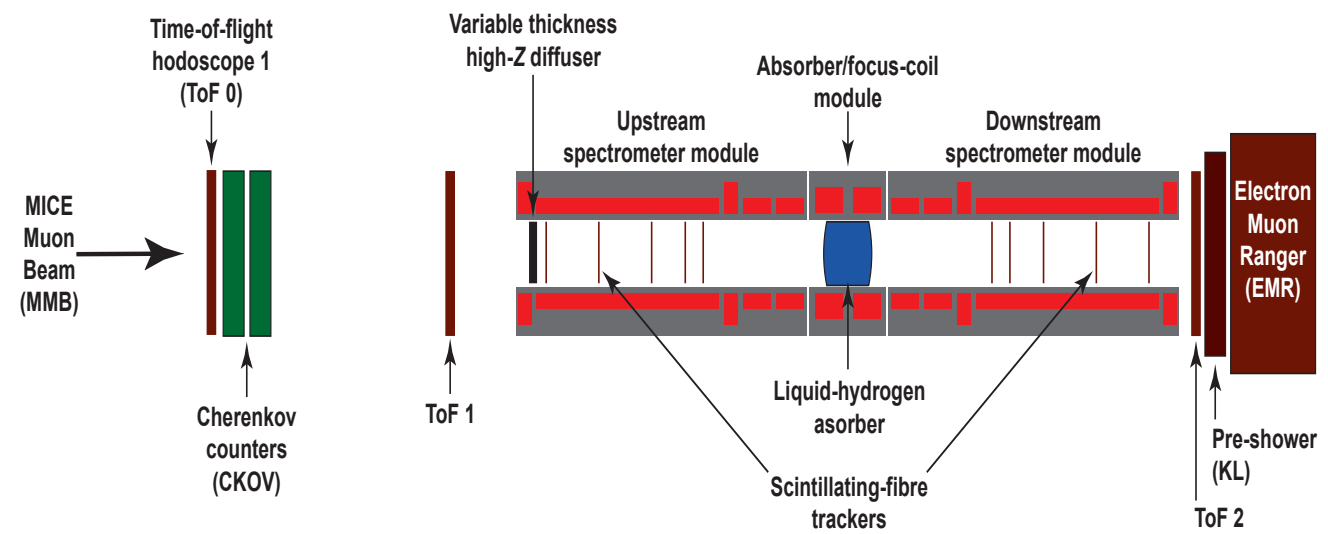

Figure 3: Schematic of Step IV of the MICE experiment, with the Absorber Focus Coil between the two Spectrometer Solenoids.

MICE will explore the parameter space using several low-Z absorbers, including liquid hydrogen, at a variety of $\beta_{\perp}$ functions.

\section{MICE Beam Line and Experiment}

The MICE experiment is located at the Rutherford Appleton Laboratory (RAL) in the UK and operates parasitically on the ISIS proton accelerator [4], producing beam for the newly built MICE Muon Beam (MMB) by the insertion of an internal pion-production target. MICE is a novel single particle experiment designed to perform high precision measurements of normalized emittance both upstream and downstream of the ionization cooling equipment. A schematic of the MMB at RAL is shown in figure 2. The MMB is composed of three quadrupole triplets, two dipole magnets, which select the momentum, and a decay solenoid (DS), which increases the number of muons in the beam. A range of beam emittances can be produced immediately in front of MICE via multiple Coulomb scattering in a high-Z diffuser. When running positive beams a 
proton absorber (PA) composed of borated polyethylene is inserted into the beam line to reduce the proton flux in the downstream beam line. The MICE Step IV setup is shown in figure 3. It consists of an Absorber Focus Coil (AFC) located between two measurement stations. These stations are composed of particle identification suites including a total of three time-of-flight detectors (TOFs), two Cherenkov detectors (Ckova and Ckovb), the KLOE-type sampling calorimeter (KL) [5] and the Electron Muon Ranger (EMR). Each station has a Tracker with five planes of scintillating fibres inside a $4 \mathrm{~T}$ Spectrometer Solenoid (SS) to measure track and momentum information $\left(x, y, p_{x}\right.$ and $p_{y}$ ), so as to reconstruct the emittance before and after cooling.

\subsection{Diffuser}
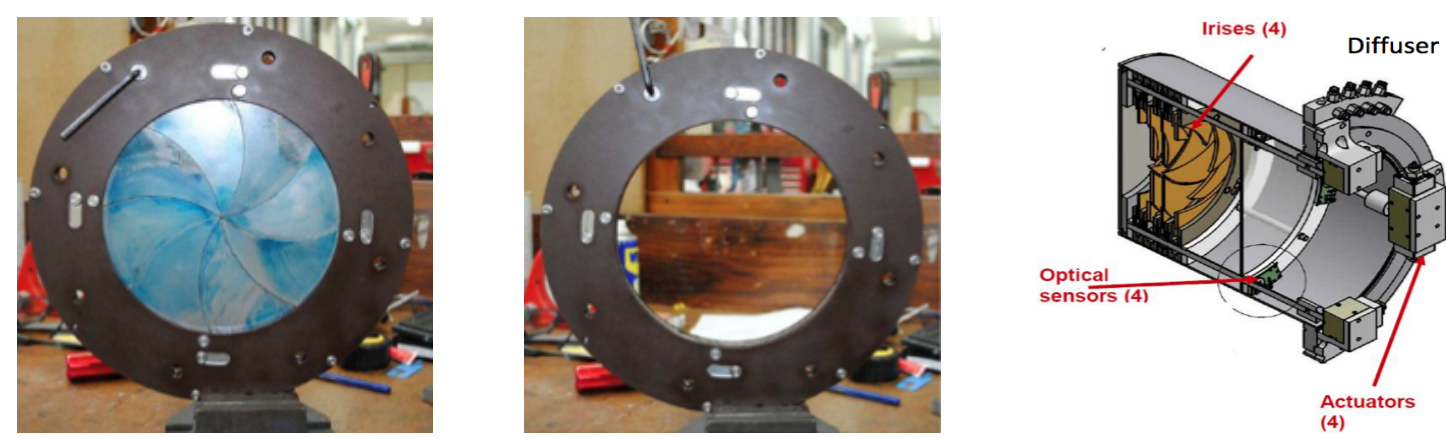

Figure 4: The MICE diffuser with iris closed (left), iris open (centre) and cross-section (right).

The diffuser (figure 4) will be used to vary the emittance of the incoming beam. It consists of four in-line irises contained in cassettes and stacked in a drum. These are located on the upstream face of the upstream SS body and the irises can be opened and closed remotely in various configurations. Commissioning of the diffuser, determining the configuration of irises required for each setting, will be a core activity within the beam line commissioning task.

\section{Step IV}

In MICE Step IV an AFC module, which will house the liquid hydrogen or solid absorber within a focusing coil, is placed between the two measurement stations. A pair of match coils in each spectrometer tune the magnetic optics to match the muon beam into and out of the AFC. Following the completion of Step IV, RF cavities will be installed for the demonstration of ionization cooling.

The currents in the matching coils and in the focus coils will vary considerably for different momentum and optical settings. In addition to various momentum and optical settings, MICE can be operated in both 'flip' and 'solenoid' modes. By comparing the transmitted distributions between the two modes it is possible to examine the behavior of the beam envelope and canonical angular momentum. Each beam delivered is designed to be matched into the upstream tracking volume, satisfying $\alpha_{\perp}=0, \beta_{\perp}=333 \mathrm{~mm}$ (figure 5) at $p_{z}=200 \mathrm{MeV} / \mathrm{c}$. These conditions define the matching conditions for MICE. Matching into and out of the AFC will be examined by altering the tuning of the lattice and measuring the beams optical parameters. 


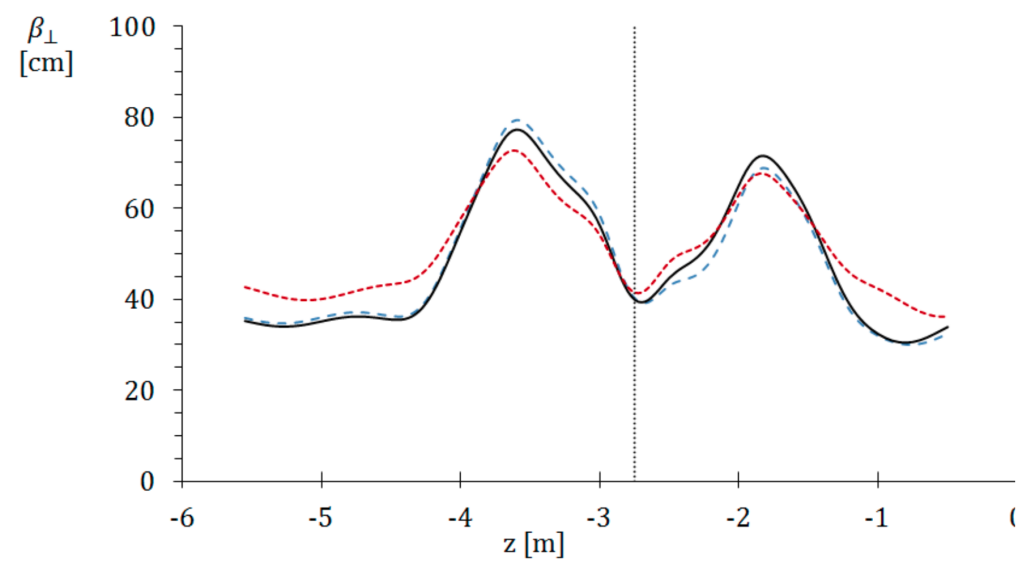

Figure 5: MAUS simulation of Beta function in MICE Step IV for $240 \mathrm{MeV} / \mathrm{c}$ (red), $200 \mathrm{MeV} / \mathrm{c}$ (black) \& $140 \mathrm{MeV} / \mathrm{c}$ (blue). The vertical dashed line indicates the centre of the absorber. [[6]].

\section{Beam Commissioning}

The commissioning of MICE has several objectives and will proceed through various steps which will now be outlined.

\subsection{Objectives for Beam Commissioning}

- Verify the optical properties of the MMB;

- Verify the matching conditions into the AFC including the effect of the diffuser that will allow the manipulation of the value of the input emittance.

\subsection{Commissioning Block}

Table 1 shows a 'physics block' in the MICE program. The parameter space in emittance, $\varepsilon_{N}$, and momentum, $p_{z}$, will be explored with beams of various $\beta_{\perp}$ functions. A 'physics block' assumes data taking at each of three momenta and three different input emittance settings. This requires that nine matching conditions be identified.

\subsection{Beam Line Pre-Commissioning}

Before the main commissioning activities take place a number of tasks must be completed. A dedicated run for Tracker alignment without $B$ fields in the spectrometer solenoids will be required and a shakedown of the beam line hardware must be performed. At this point the new MMB settings required for Step IV operations with the diffuser can be tested. The tomography-like phase-space reconstruction developed and tested in Step I operations [7] will allow the matching condition at TOF0 to be measured and compared with Monte Carlo predictions for the new settings. It will be necessary to collect $\sim 10 \mathrm{k}$ good triggers per setting ( $\sim 8$ shifts for all settings). A particle trigger is defined as a coincidence of the PMTs at the two ends of the same scintillation counter in the TOF1 station [[4]]. Logic units combine this signal with a signal from the DAQ system generated after the extraction of the ISIS proton beam, this combined signal defines a good particle trigger. 
Table 1: $\left(\varepsilon_{N}, p_{z}\right)$ matrix for the MICE programme. The Twiss parameters $\left(\alpha_{\perp}, \beta_{\perp}\right)$ are those required at the upstream face of the diffuser of thickness $d$ for a matched beam [[4]].

\begin{tabular}{|c|c|c|c|c|}
\hline & \multicolumn{3}{|c|}{$p_{z}(\mathrm{MeV} / \mathrm{c})$} \\
\hline & & 140 & 200 & 240 \\
\hline \multirow{12}{*}{ 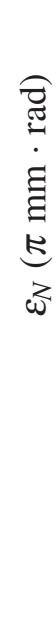 } & & $d=0.0$ & $d=0.0$ & $d=0.0$ \\
\hline & 3 & $p_{\text {diff }}=151 \mathrm{MeV} / \mathrm{c}$ & $p_{\text {diff }}=207 \mathrm{MeV} / \mathrm{c}$ & $p_{\text {diff }}=245 \mathrm{MeV} / \mathrm{c}$ \\
\hline & 3 & $\alpha_{\perp}=0.2$ & $\alpha_{\perp}=0.1$ & $\alpha_{\perp}=0.1$ \\
\hline & & $\beta_{\perp}=56 \mathrm{~cm}$ & $\beta_{\perp}=36 \mathrm{~cm}$ & $\beta_{\perp}=42 \mathrm{~cm}$ \\
\hline & & $d=0.9$ & $d=1.3$ & $d=1.3$ \\
\hline & 6 & $p_{\text {diff }}=156 \mathrm{MeV} / \mathrm{c}$ & $p_{\text {diff }}=215 \mathrm{MeV} / \mathrm{c}$ & $p_{\text {diff }}=256 \mathrm{MeV} / \mathrm{c}$ \\
\hline & 0 & $\alpha_{\perp}=0.3$ & $\alpha_{\perp}=0.2$ & $\alpha_{\perp}=0.2$ \\
\hline & & $\beta_{\perp}=113 \mathrm{~cm}$ & $\beta_{\perp}=78 \mathrm{~cm}$ & $\beta_{\perp}=80 \mathrm{~cm}$ \\
\hline & & $d=1.8$ & $d=2.8$ & $d=2.8$ \\
\hline & 10 & $p_{\text {diff }}=164 \mathrm{MeV} / \mathrm{c}$ & $p_{\text {diff }}=229 \mathrm{MeV} / \mathrm{c}$ & $p_{\text {diff }}=267 \mathrm{MeV} / \mathrm{c}$ \\
\hline & & $\alpha_{\perp}=0.6$ & $\alpha_{\perp}=0.4$ & $\alpha_{\perp}=0.3$ \\
\hline & & $\beta_{\perp}=198 \mathrm{~cm}$ & $\beta_{\perp}=131 \mathrm{~cm}$ & $\beta_{\perp}=129 \mathrm{~cm}$ \\
\hline
\end{tabular}

\subsection{Beam Line Commissioning}

The main objective of this activity will be to test the MMB matching conditions. This will require that the DS, proton absorber, all of the beam line magnets, TOF0 and TOF1, the diffuser and the upstream Tracker (which requires $B$ field in the upstream SS) are all commissioned. The 9 settings of the beam matrix (table 1 ), each require $\sim 10 \mathrm{k}$ triggers which equates to $\sim 10 \mathrm{~h}$ of useful beam. It is therefore estimated that 15 shifts (including contingency) will be required in order to complete this activity.

\subsection{Beam Commissioning for Step IV}

The trajectory of particles through MICE will be measured with the Trackers with the mean radial position and divergence expected to be close to zero. The $\alpha_{\perp}, \beta_{\perp}$ and emittance of the beam will be measured at all 10 tracker planes and with this information the transfer matrix through MICE can be calculated and the predicted evolution of the $\beta_{\perp}$ function measured. At this point the observed behavior can be compared with the Monte Carlo prediction potentially allowing for retuning of the magnet settings. Including any additional time for calibration runs, extra tuning and including contingency the estimated number of shifts is $\sim 21$ for assessing all three momentum settings.

\section{G4Beamline Simulations}

G4beamline is a particle tracking simulation program based on Geant4. It is optimised for simulating beam lines, and is especially useful for muon facilities in which decays and/or interactions with matter are important [8]. The MMB is simulated using this package with VirtualDetector planes placed every $10 \mathrm{~cm}$ from the centre of the DS to TOF1. A VirtualDetector generates full tracking information for each track when it enters its physical volume, which is set to vacuum and 

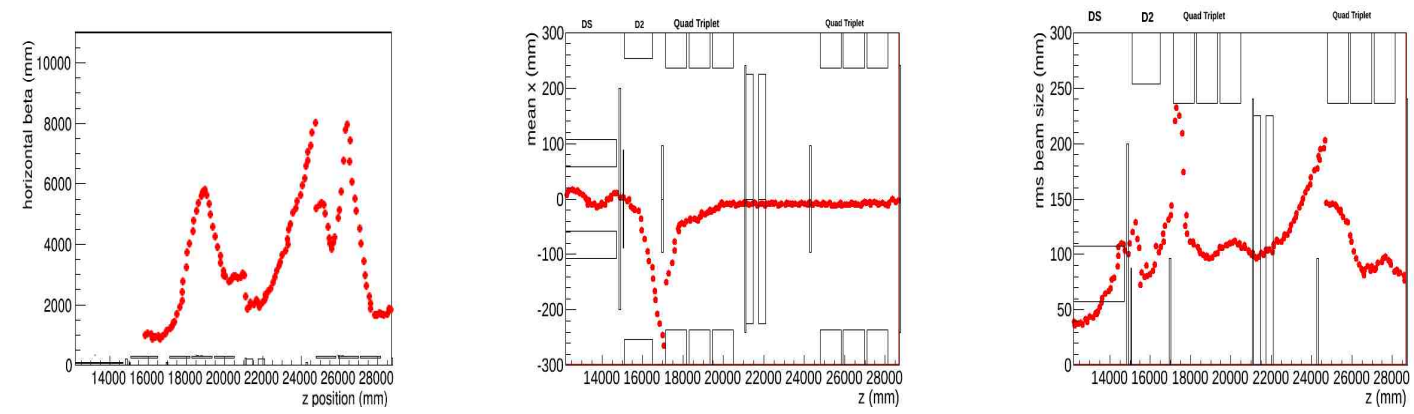

Figure 6: $2 \mathrm{D}$ distributions of $\beta_{x}$, mean position and RMS beam size (left, center and right respectively) as a function of position along the MMB for a $6 \pi \mathrm{mmrad}, 140 \mathrm{MeV} / \mathrm{c} \mu^{+}$beam in $x$. Distances are with respect to the MICE target.
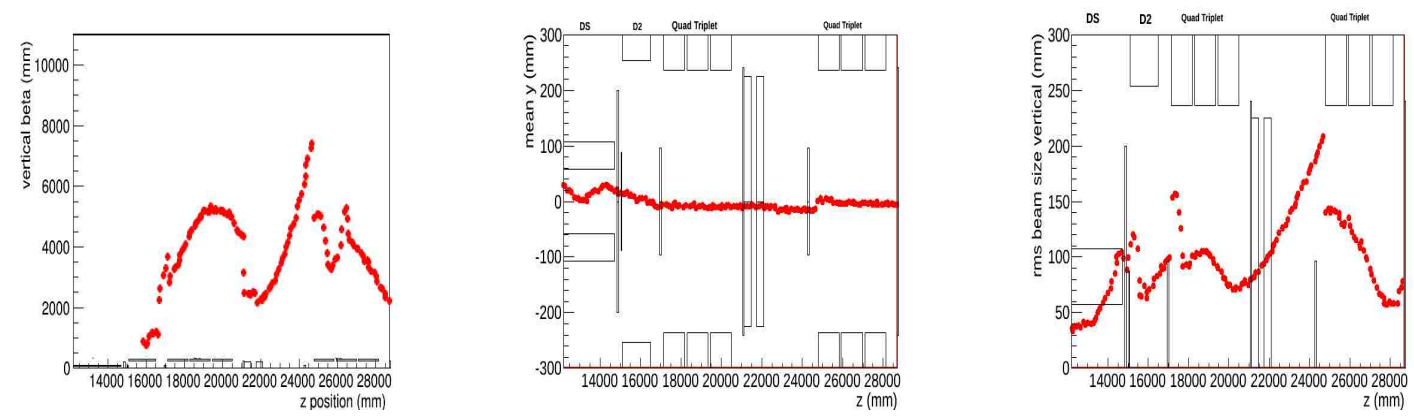

Figure 7: 2D distributions of $\beta_{y}$, mean position and RMS beam size (left, center and right respectively) as a function of position along the MMB for a $6 \pi \mathrm{mmrad}, 140 \mathrm{MeV} / \mathrm{c} \mu^{+}$beam in $y$. Distances are with respect to the MICE target.

does not disturb the particle crossing it. This allows various beam parameters to be calculated at every plane and plotted as a function of $Z$ (where $Z$ is oriented along the direction of the MMB). In this way the behavior and matching of the beam in the MMB can be understood.

Tracking beam parameters along the beam line allows the beam line settings to be optimised. The focusing of the beam in both orientations (figure 6,7 ) can be cross-checked and the behavior in $\mathrm{Z}$ inspected with these visual tools.

\subsection{Plan for Beam Optimisation}

Starting from a description of a beam including all materials and solenoidal fields a prediction of the settings for the MMB can be made. Here the optics of the magnets together with the matter effects of TOF0, TOFI and the diffuser materials are taken into account. The MMB must be matched closely to the uniform field of the Spectrometer Solenoid in the Tracker region (4T) with $\alpha=0$ to deliver the required performance for MICE. From the discontinuities in figure 6 and 7 it is clear that the first pass settings are not providing matched beam into MICE. Using these setting as a starting point the commissioning process can optimise the settings with information gained from the Monte Carlo simulations and verify them with the data collected during the commissioning process. 


\begin{tabular}{|c|c|}
\hline Beam commissioning phase & Number of $8 \mathrm{~h}$ sifts \\
\hline Beam line pre-commissioning & 8 \\
Beam line commissioning & 15 \\
Mice Channel commissioning & 21 \\
\hline
\end{tabular}

Figure 8: Summary of shifts required to complete MICE Step IV commissioning.

\section{Conclusion}

The procedure for commissioning the MMB and MICE experiment at Step IV has been outlined. The time and number of shift required to complete this work has been calculated. G4beamline simulations of the evolution of beam parameters through the beam line are ready. These results will be used in the commissioning process to identify the optimal settings for MICE.

\section{References}

[1] Y. Fukuda et al. Evidence for oscillation of atmospheric neutrinos. Phys.Rev.Lett., 81:1562-1567, 1998.

[2] J. Farine. Measurement of the rate of nu/e $+d \rightarrow p+p+e-$ interactions produced by B- 8 solar neutrinos at the Sudbury Neutrino Observatory. Phys.Atom.Nucl., 65:2147-2155, 2002.

[3] S. Geer. Neutrino beams from muon storage rings: Characteristics and physics potential. Phys.Rev, D57:6989-6997, 1998.

[4] M. Bogomilov et al. The MICE Muon Beam on ISIS and the beam-line instrumentation of the Muon Ionization Cooling Experiment. JINST, 7:P05009, 2012.

[5] M. Adinolfi, A. Aloisio, F. Ambrosino, A. Andryakov, A. Antonelli, et al. Calibration and reconstruction performances of the KLOE electromagnetic calorimeter. Nucl.Instrum.Meth., A461:344-347, 2001.

[6] Timothy Carlisle. Step IV of the Muon Ionization Cooling Experiment (MICE) and the Multiple Scattering of Muons. PhD thesis, University of Oxford, 2013.

[7] D. Adams et al. Characterisation of the muon beams for the Muon Ionisation Cooling Experiment. Eur.Phys.J., C73:2582, 2013.

[8] T. Roberts et al. G4beamline, A "Swiss Army Knife" for Geant4, optimized for simulating beamlines. http://www. muonsinc.com/muons3/G4beamline, 2013. 